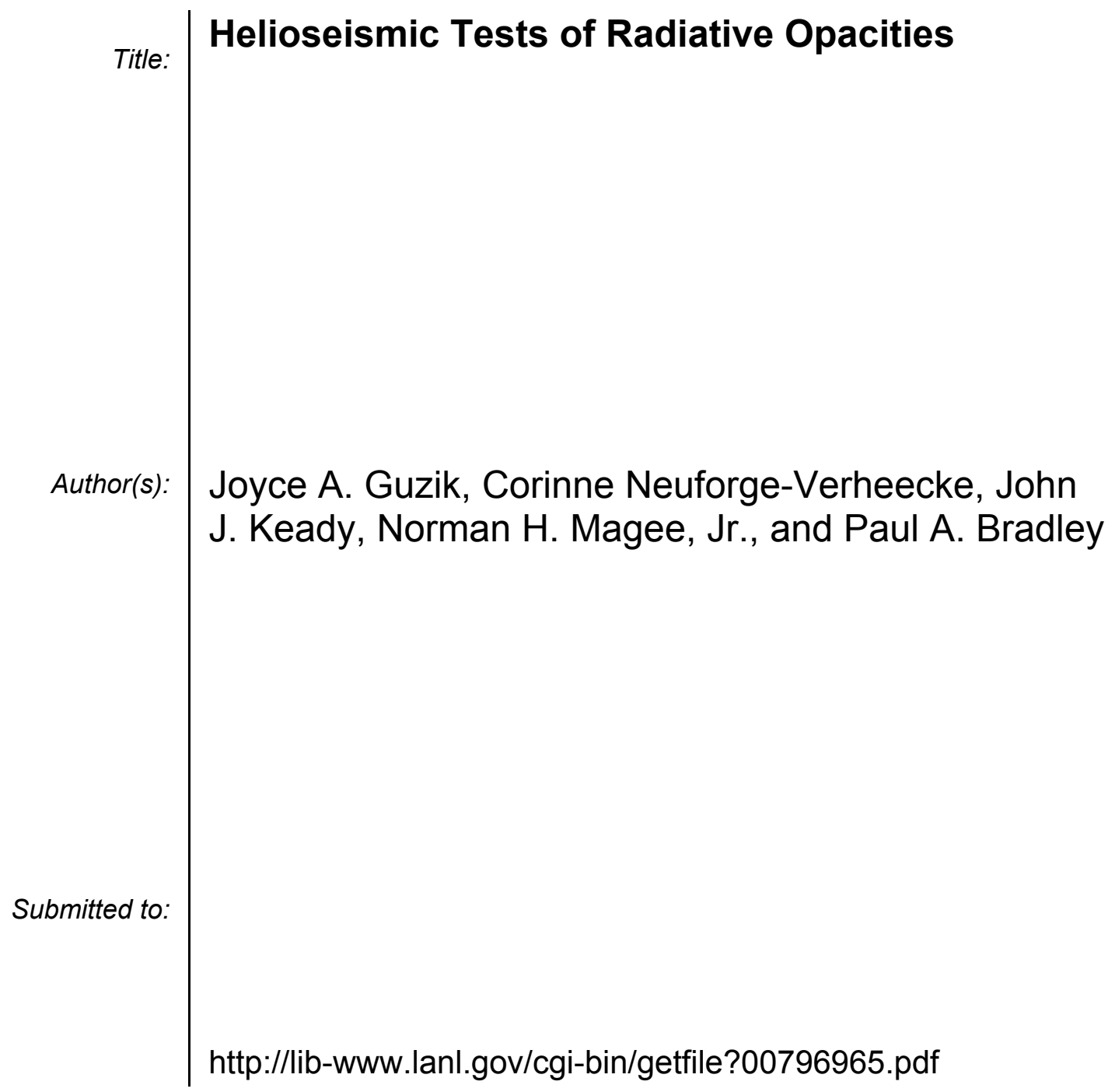




\title{
Helioseismic Tests of Radiative Opacities
}

\author{
Joyce A. Guzik, Corinne Neuforge-Verheecke, John J. Keady, \\ Norman H. Magee, Jr., and Paul A. Bradley
}

Los Alamos National Laboratory

Los Alamos, NM 87545-2345 USA

\begin{abstract}
During the past fifteen years, thousands of solar acoustic oscillation modes have been measured to remarkable precision, in many cases to within $0.01 \%$. These frequencies have been used to infer the interior structure of the sun and test the physical input to solar models. Here we summarize the procedures, input physics and assumptions for calculating a standard solar evolution model. We compare the observed and calculated sound speed profile and oscillation frequencies of solar models calibrated using the new Los Alamos LEDCOP [1] and Livermore OPAL [2] Rosseland mean opacities for the same element mixture. We show that solar oscillations are extremely sensitive to opacities, with opacity differences of only a few percent producing an easily detectable effect on the sound speed and predicted frequencies [3]. The oscillation data indicate that agreement would be improved by an opacity increase of several percent below the convection zone for both the LEDCOP and OPAL opacities.
\end{abstract}

\section{INTRODUCTION}

During the past 15 years, the frequencies of millions of global solar acoustic modes of oscillation have been measured to high precision, in some cases to within a few parts in a million. These modes are stochastically excited by turbulence in the upper part of the convective envelope and are called pressure modes ( $p$-modes) because the restoring force involved in the oscillation is due to the pressure gradient. The Sun acts as an acoustic cavity that traps these waves, and modes of different frequencies and angular degree penetrate to different depths in the solar interior. Since the frequencies depend on the interior sound speed gradient, they can be used to probe solar structure. Using solar oscillation frequencies to infer the structure of the Sun and to test the physical input used in solar models is called "helioseismology". For additional information, see the review articles in the May 31, 1996 issue of Science [4].

The conditions in the solar interior, where radiative diffusion transports energy, span the temperature and density range from 15.6 million $\mathrm{K}(1.3 \mathrm{keV})$ and $150 \mathrm{~g} \mathrm{~cm}^{-3}$ in the solar center, to 2.2 million $\mathrm{K}(0.19 \mathrm{keV})$ and $0.2 \mathrm{~g} \mathrm{~cm}^{-3}$ at the base of the convection zone. The radiative opacity is crucial to determine the structure of the Sun and the frequencies of the acoustic modes, since the opacity determines the temperature gradient, and hence affects the sound-speed gradient. With increasing opacity toward the solar envelope, convection becomes a more efficient means of energy transport than radiative diffusion. In the Sun's convective envelope, comprising the outer 30 percent of the radius, the temperature gradient becomes nearly adiabatic, and the sound speed is instead determined entirely by the mean molecular 
weight and the efficiency of convective energy transport. Near the solar surface, at temperatures of $12,000 \mathrm{~K}$ or less, radiative opacities, including molecular opacities, once again become important. Helioseismic frequency inversion techniques, used to determine the Sun's interior structure, have been developed to eliminate sensitivity to uncertainties in the near-surface structure.

Here we summarize evolution modeling procedures and physics of standard solar models. We then compare two solar models: one calculated with the Livermore OPAL opacities [2], and one calculated with the recent Los Alamos LEDCOP (Light Element Detailed Configuration Opacity) opacities [1], in light of the helioseismic data and inferences (oscillation frequencies, sound speed profile and location of the base of the convection zone). We discuss the reasons for the differences between the OPAL and the LEDCOP opacities. Finally, we comment on the sensitivity of solar structure to other input physics or sources of uncertainty, and the prospects for inferring solar interior opacities from helioseismic data.

\section{SOLAR EVOLUTION MODELING}

To obtain a calibrated model for the present Sun, we solve the equations of stellar structure for a sequence of time steps from the onset of the nuclear reactions to the present solar age. The equations that are solved include those for mass conservation; hydrostatic equilibrium; energy production and loss; and energy transport from the interior to the surface via radiative diffusion, electron conduction, or convection. These one-dimensional models are calculated assuming spherical symmetry, negligible mass loss or mass accretion; no rotation or magnetic fields; an initially homogeneous chemical composition; and assuming that the Sun's luminosity is generated mainly by conversion of hydrogen to helium via fusion reactions in the core. Modern solar models also include diffusive settling of helium and heavier elements relative to hydrogen. This diffusion is very slow, and impeded by the convective mixing in the envelope; however, during the Sun's 4.5 billion-year lifetime, about 10 percent of the initial helium and $\sim 8$ percent of the surface heavier elements can settle from the convection zone. This diffusion has a significant effect on the Sun's structure (see, e.g., [5], [6], [26]).

Solving the equations requires physical data, including: radiative and conductive opacities (as a function of temperature, density, and element composition); an equation of state, giving the pressure, energy, and other thermodynamic quantities as a function of temperature, density, and composition; nuclear reaction rates; element diffusion coefficients; and a treatment for convective energy transport.

Calculating a solar model is an iterative process. We typically divide the model into several hundred mass shells from the center to the surface, and the evolution into several hundred time steps. One begins by adopting an initial helium mass fraction $\left(\mathrm{Y}_{\mathrm{o}}\right)$, initial mass fraction of elements heavier than $\mathrm{H}$ and $\mathrm{He}\left(\mathrm{Z}_{\mathrm{o}}\right)$, and an initial guess for the ratio of the mixing length to pressure scale height, $\square$, that regulates the convective efficiency. $Y_{0}, Z_{0}$, and $\square$ are adjusted so that the evolved model reaches the observed luminosity Ls, radius Rs, and present surface ratio of heavy element to 
hydrogen mass fraction, $\mathrm{Z} / \mathrm{X}$ at the present solar age. Because of diffusion, the present surface $\mathrm{Z} / \mathrm{X}$ is not equal to $\mathrm{Z}_{\mathrm{o}} / \mathrm{X}_{0}$.

The present and assumed constant mass of the Sun is $1.989110^{33} \mathrm{~g}$ [7]. The Sun does lose some mass via the solar wind, but only at the negligible rate of $210^{-14} \mathrm{Ms}$ per year. We adopt Rs $=6.959910^{10} \mathrm{~cm}, \mathrm{Ls}=3.84610^{33} \mathrm{erg} \mathrm{s}^{-1}$, and the solar age determination of $4.52 \pm 0.04 \mathrm{Gyr}$ [8]. We adopt the present $\mathrm{Z} / \mathrm{X}=0.0245$ and element mixture from the Grevesse \& Noels 1993 solar element abundance determination [9].

We evolve our models using an extensively updated version of the Iben code [10], described in more detail in [11] and [12]. The code includes the treatment of Burgers [13] to calculate the thermal, gravitational and chemical diffusion of the electrons and 9 additional isotopes of $\mathrm{H}, \mathrm{He}, \mathrm{C}, \mathrm{N}, \mathrm{O}, \mathrm{Ne}$ and $\mathrm{Mg}$ (see [5] for details). We smoothly join the opacities that we use in the solar interior (either OPAL or LEDCOP) to the low-temperature tables of Alexander \& Ferguson [14] used at the surface by a sinusoidal average between $7500 \mathrm{~K}$ and $9500 \mathrm{~K}$. Our convection treatment is the standard mixing length theory [15]. We adopted the SIREFF analytical equation of state [11]. All charged-particle nuclear reactions are taken from Angulo et al. [16]. See [3] and [12] for additional details on the nuclear reaction rate calculations.

\section{HELIOSEISMIC COMPARISONS}

Table 1 summarizes the properties of our two evolution models that are calibrated to the present solar luminosity, radius, and $\mathrm{Z} / \mathrm{X}$, using either the OPAL or LEDCOP opacities.

TABLE 1. Properties of Calibrated Solar Evolution Models.

\begin{tabular}{lcc}
\hline & $\begin{array}{c}\text { OPAL Opacity } \\
\text { Model }\end{array}$ & LEDCOP Opacity Model \\
\hline Initial H mass fraction $\mathrm{X}_{\mathrm{o}}$ & 0.7100 & 0.7122 \\
Initial He mass fraction $\mathrm{Y}_{\mathrm{o}}$ & 0.2703 & 0.2680 \\
Initial element mass fraction $\mathrm{Z}_{\mathrm{o}}$ & 0.0197 & 0.0198 \\
Mixing Length/Pressure scale height $(\square)$ & 1.7738 & 1.7651 \\
& & \\
$\mathrm{~T}_{\text {central }}\left(10^{6} \mathrm{~K}\right)$ & 15.66 & 15.66 \\
$\mathrm{C}_{\text {central }}\left(\mathrm{g} \mathrm{cm}^{-3}\right)$ & 152.2 & 150.8 \\
$\mathrm{Y}_{\text {central }}$ & 0.6375 & 0.6350 \\
$\mathrm{Z}_{\text {central }}$ & 0.0208 & 0.0209 \\
$\mathrm{R}_{\text {convection zone base }}(\mathrm{R} / \mathrm{Rs})$ & & \\
$\mathrm{T}_{\text {convection zone base }}\left(10^{6} \mathrm{~K}\right)$ & 0.7135 & 0.7177 \\
$\mathrm{Y}_{\text {convection zone }}$ & 2.195 & 2.148 \\
\hline
\end{tabular}

Figure 1 compares the opacity differences as a function of radius for the two models. The convection zone of the LEDCOP model is shallower (see Table 1) due to the fact that the Los Alamos opacities are up to 6\% lower than the OPAL opacities near the base of the convection zone, as can be seen in Figure 1. The OPAL model has a convection zone base location in better agreement with the helioseismic inference of $\mathrm{R} / \mathrm{Rs}=0.713 \pm 0.001$ [17]. The two models have slightly different 
composition, density and temperature profiles due to the small adjustments in composition and the mixing-length parameter required to calibrate the model to current solar conditions, and the opacity difference at a given radius is reduced by this calibration. The absolute differences between OPAL and LEDCOP opacities for the same density, composition, and temperature profiles are only slightly higher (up to $2 \%$ ) than seen in Fig. 1 for a portion of the solar interior (see Fig. 1 of [3]).

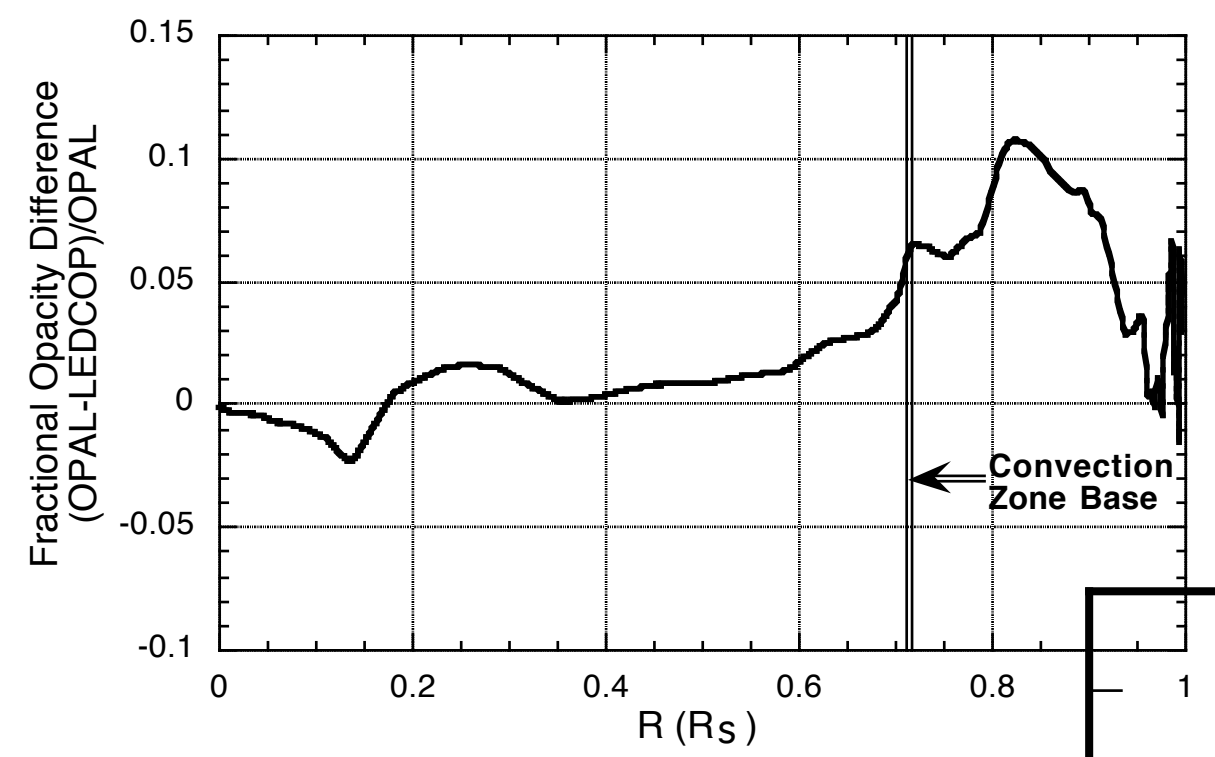

FIGURE 1. Relative opacity difference vs. fractional solar radius for two calibrated solar models using OPAL and LEDCOP opacities. The vertical lines indicate the convection zone base location.

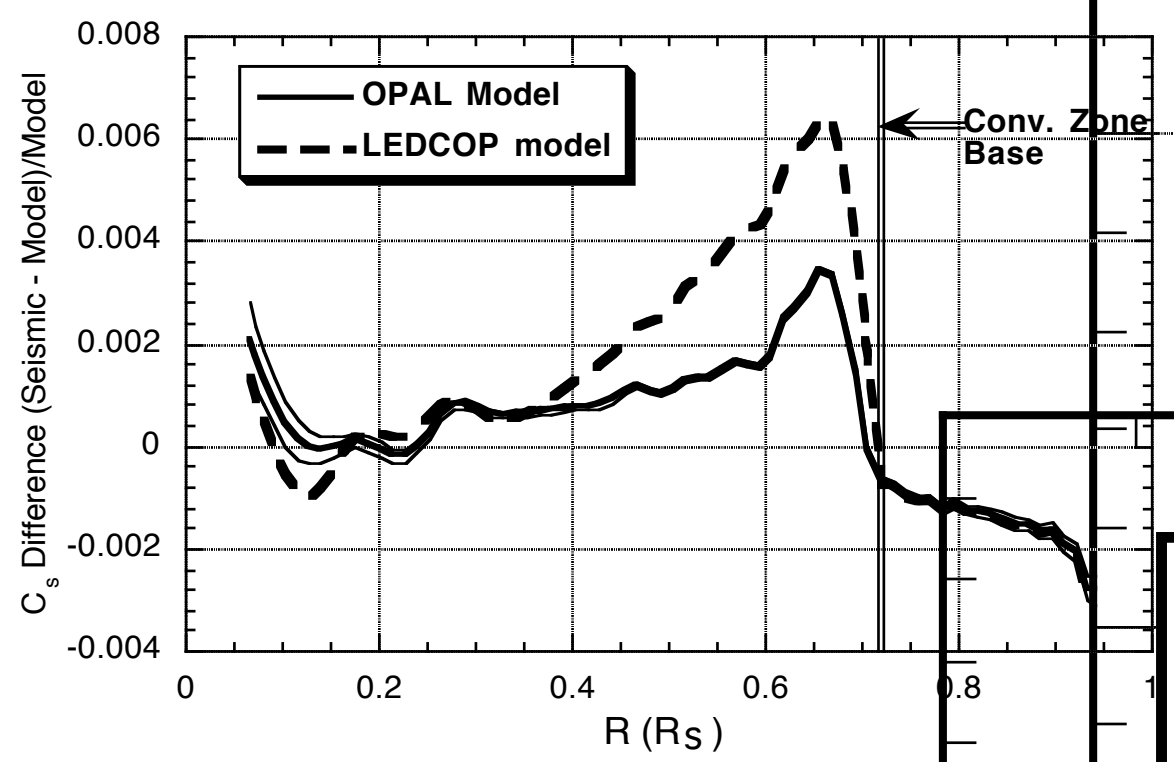

FIGURE 2. Relative sound speed differences between two solar models and the seismic inversion [18], $\left(\mathrm{c}_{\text {seismic }}-\mathrm{c}_{\text {model }}\right) / \mathrm{c}_{\text {model }}$, as a function of the fractional radius $\mathrm{R} /$ Rs. The errors on the inversion are 
shown for the OPAL model curve, and are approximately as large as the line width except near the solar center. The vertical lines indicate the convection zone base location.

Figure 2 compares the sound speed differences between the models and the sound speed profile inferred from helioseismic inversions by Basu et al. [18]. The error bars on the inversion are quite small compared to the differences between the model and inferred sound speed, indicating that significant improvement is necessary. However, note that the sound speed profiles of these models agree with the inferred sound speed to within a fraction of a percent. The agreement is better for the OPAL model from the base of the convection zone to $\mathrm{R} / \mathrm{Rs}=0.12$.

We use the linear non-adiabatic pulsation code of Pesnell [19] to calculate the pmode oscillation spectrum of our models. A solar oscillation mode can be characterized by three numbers: the radial order $n$, giving the number of radial nodes in the eigenfunction; the angular degree $\square$, giving the number of node lines on the surface; and the azimuthal order $m$, giving the number of node lines through the pole when spherical symmetry is broken, e.g. by rotation. Higher-order modes have higher frequencies and penetrate less deeply. Also, low-degree modes penetrate deeper into the Sun than do high-degree modes. For the low-degree frequency comparisons $(\square=0$, $1,2,3)$, we use a hybrid set of observational data described in $[3,12]$ chosen to maximize the number of observed low-degree modes in the set and minimize the observational uncertainties. For the intermediate-degree frequency comparisons, we use the data from [20].

Figure 4 shows observed minus calculated (O-C) nonadiabatic frequency differences $(\mu \mathrm{Hz})$ vs. calculated frequency $(\mu \mathrm{Hz})$ for some low-degree $(\square=0,1,2$ and $3)$ and intermediate-degree $(\square=5,10,15$ and 20) p-modes from our calibrated models. The turning points for these modes are well below the convection zone base. Note first that the agreement is excellent, with the differences no larger than a few $\mu \mathrm{Hz}$ out of a few thousand, or within $0.1 \%$. The observational uncertainties for these modes are less than $0.1 \mu \mathrm{Hz}$, so that the trends in the frequency differences are significant. The reasons for the trends have been investigated (see, e.g., [20]). The upward trend in O-C frequency at low frequency can be removed [21] by decreasing the adopted value for the present solar radius by about $400 \mathrm{~km}$, as was recently derived [22]. The downward trend at higher frequencies can be removed by a very slight adjustment to the sound speed gradient at the top of the solar convection zone, between 9,000 and $12,000 \mathrm{~K}$, obtainable by improving the convection treatment and including turbulent pressure effects. An overall upward or downward shift in $\mathrm{O}-\mathrm{C}$ frequency for these low and intermediate-degree modes with turning points below the convection zone base can be induced by changing the convection zone depth. The O-C frequencies of the LEDCOP model are generally higher than those of the OPAL model because the convection zone is shallower, and further from the inferred value of $0.713 \mathrm{Rs}$. The dispersion in frequency difference as a function of degree is also larger for the LEDCOP model. The modes of higher $\square$ penetrate more deeply, and are therefore sensitive to different integrated regions of the Sun from the surface to the turning point. The dispersion therefore reflects the larger difference between the model and actual sound speed gradient below the convection zone evident in Fig. 2. 


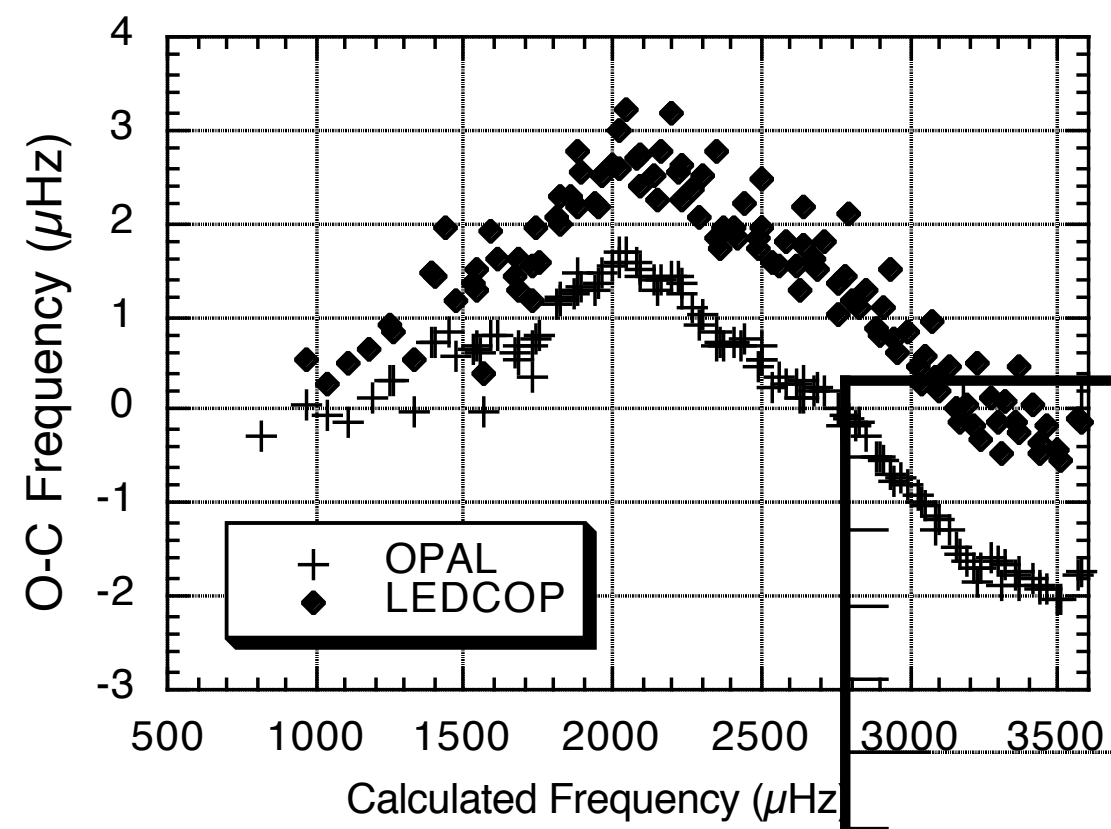

FIGURE 3. Observed minus calculated $(\mathrm{O}-\mathrm{C})$ nonadiabatic frequency differences $(\mu \mathrm{Hz})$ vs. calculated frequency $(\mu \mathrm{Hz})$ for low-degree $(\square=0,1,2$ and 3$)$ and intermediate-degree $(\square=5,10,15$ and 20) pmodes of calibrated OPAL and LEDCOP models.

\section{COMMENTS ON OPACITY DIFFERENCES}

The OPAL and LEDCOP opacities differ by about $6 \%$ at the base of the convection zone. We have examined the LEDCOP opacity tables to understand the reasons for the differences, and what can be done to reduce the discrepancy in future comparisons. There appear to be three causes for the differences: the opacity models, the interpolation methods, and the resolution of the temperature grids used by the two tables.

Little information about the opacity models can be extracted from the astrophysical opacity tables, since they contain information for almost 30 elements, which has been integrated over frequency and then has been interpolated in $\mathrm{R}\left(=\square / \mathrm{T}^{3}\right.$, where $\mathrm{T}$ is in units of $10^{6} \mathrm{~K}$ ). Fortunately, there have been a series of opacity workshops [23], in which OPAL, LEDCOP, and many other opacity codes have been compared in detail. At the latest meeting [24], the opacities for the Grevesse and Noels 1993 element mixture were compared for solar center conditions. The OPAL results were $3 \%$ higher than LEDCOP. There are no comparisons at the physical conditions of the convection zone base, but some pure element cases, mainly iron and carbon, are relatively close to these physical conditions $\left(\mathrm{T}=210^{6} \mathrm{~K}, \log \mathrm{R}=-1.5\right)$.

Concentrating principally on iron and taking into account the relative contributions to the mixture from the individual elements, we estimate that the LEDCOP opacities intrinsically are $2.5 \pm 2 \%$ lower than OPAL for this $(\mathrm{T}, \mathrm{R})$ regime. We believe that this is due to differences in line transition energies, different level abundances obtained by the two equations of state, and continuum lowering models and treatment of far line wings, especially for the H-like and He-like Stark profiles. 
The pure element LEDCOP opacities are calculated on a temperature and chemical potential grid, which allows the elements to be combined into mixtures. This table is then linearly interpolated in density to the final T-R astrophysical table grid. A spline interpolation is then used to obtain opacities for all $\mathrm{X}, \mathrm{Z}, \mathrm{T}$ and $\mathrm{R}$. The spline interpolation has been checked and is able to reproduce the tabular values to $\sim 1 \%$. When the interpolated opacity at the convection zone base was compared with a direct opacity calculation by LEDCOP, the interpolated value was 3.5\% lower than the actual calculation, with an uncertainty of $1 \%$ due to the spline interpolation. Independent comparisons for oxygen confirm that the linear interpolation routines produce values that are 4 to $5 \%$ low for oxygen in this region of the T-R table. Note that oxygen is the most important contributor to the opacity at the base of the solar convection zone [25].

A final source of discrepancy is due to the different logarithmic temperature grids used by OPAL and LEDCOP. Each table has 10 temperatures per decade, but with different spacing. The LEDCOP table does not have an opacity value near $210^{6} \mathrm{~K}$ and $\log \mathrm{R}=-1.5$, whereas the OPAL table does (Fig. 4). This point is an inflection point in the opacity curve and without this point, the spline-interpolated opacity at the base of the convection zone is too low by $1.5 \pm 0.5 \%$.

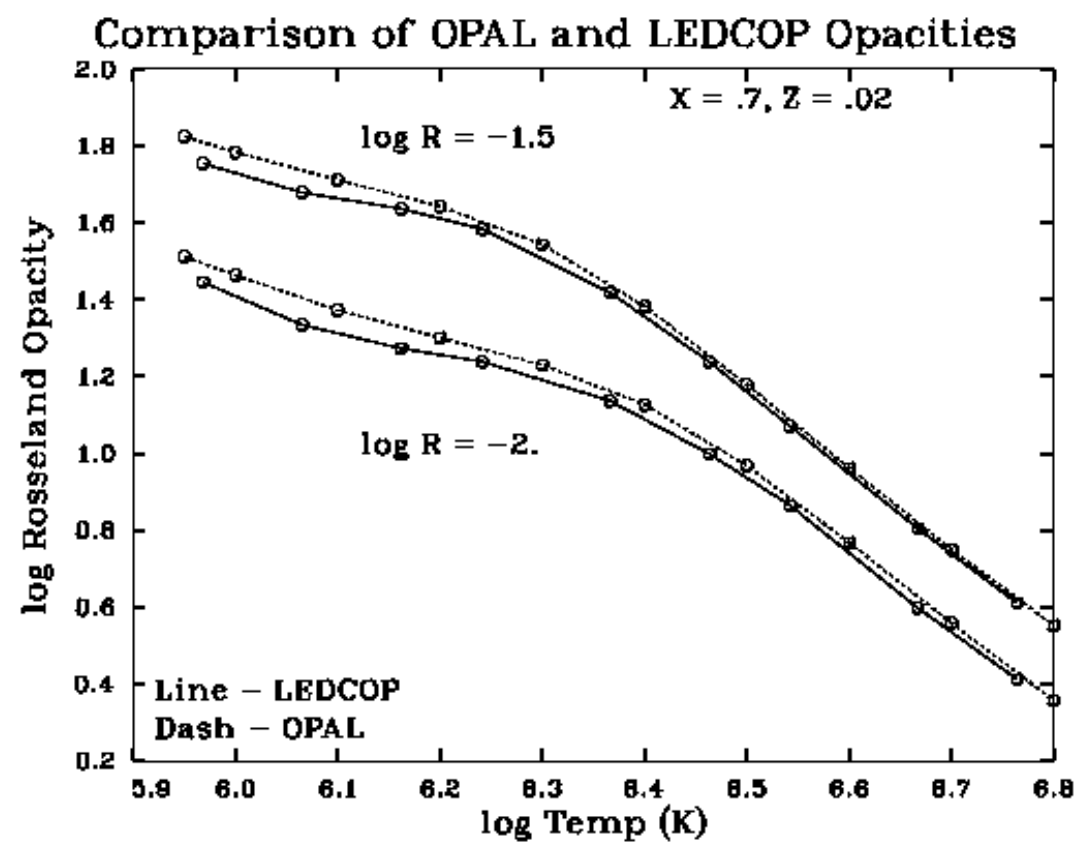

FIGURE 4. Comparison of OPAL and LEDCOP opacities for solar abundance mixture. For conditions near the solar convection zone base $(\log \mathrm{T}=6.3$ and $\log \mathrm{R}=-1.5)$, the temperature grid spacing results in a higher value for the OPAL opacity by $\sim 1.5 \%$ compared to the interpolated LEDCOP opacity.

In summary, more than half of the opacity difference between OPAL and LEDCOP at the base of the convection zone is due to interpolation errors and the choice of the temperature grid: $(3.5 \pm 1 \%)+(1.5 \pm 0.5 \%)=(5 \pm 1.5 \%)$. These problems can be reduced or eliminated by calculating more grid points for the original elemental 
calculations and using this finer mesh to produce astrophysical tables with more temperatures and at least twice as many $\mathrm{R}$ curves. There is still a fundamental difference of 2.5 to $3 \%$ between the OPAL and LEDCOP opacities at the convection zone base. Detailed OPAL vs. LEDCOP comparisons at the center of the Sun show that the opacities can vary by a few percent due to small differences in the physics choices made when generating the opacities. Therefore, it is reasonable to admit an error bar of at least $5 \%$ on the opacity calculations due to uncertainties in physical models. The fundamental difference between the OPAL and LEDCOP opacities is well within this margin of error and cannot be fully resolved until the next opacity workshop.

\section{COMMENTS ON OTHER SOURCES OF UNCERTAINTIES}

We next discuss whether the remaining small differences between the calculated and seismically inferred solar structure are due to errors in the opacities or to some other input physics or assumptions. To what precision can solar oscillations constrain opacities? How large an opacity difference is significant? As discussed above, the treatment of the solar surface layers is the reason for some of the trends in the direct frequency comparisons, but the effects of the surface layers can be removed in the inversions for the sound speed profile. We and other researchers have investigated the sensitivity of solar models to the choice of equation of state [11], nuclear reaction rates [12], and element abundances [12]. We find that the solar model structure and predicted oscillation frequencies are affected more by a few percent opacity change, for example, by switching between the LEDCOP and OPAL opacities, than they are by switching between different modern EOS treatments, by varying the abundance mixtures within the estimated uncertainties, or by varying the nuclear reaction rates within their uncertainties. The proposed reduction in solar radius of $400 \mathrm{~km}$ (see, e.g., [22]) also has a small, but non-negligible, effect on inferred sound-speed profile [17]. Note that the new solar element abundance determinations of Grevesse and Sauval [28] are lower $(\mathrm{Z} / \mathrm{X}=0.0230)$ than the Grevesse and Noels [9] abundances adopted for our models $(Z / X=0.0245)$. The new abundances result in even lower opacities at the convection zone base, and a larger discrepancy between calculated and inferred sound speed [12].

The sound speed discrepancy between models and the helioseismically inferred profile is significant at the base of the convection zone. Some of this discrepancy may be resolved by altering the diffusion-produced composition profile at the base of the convection zone, perhaps through changes in the diffusion coefficients, or mixing due to differential rotation or gravity modes (see, e.g., [6], [29]). Several studies have shown that much of the discrepancy below the convection zone can be removed by a prescribed change in the opacity profile of 1 to $\sim 5 \%[26,27]$, well within the uncertainties in opacity calculations. Calculations have been done including the effects of radiative levitation of individual elements in addition to diffusive settling; these calculations show that radiative levitation effects are quite small, amounting to a change of only $0.5 \%$ in opacity due to the different resulting element distribution, and a change of $0.06 \%$ in sound speed below the convection zone [30]. With a better 
understanding of potential mixing and diffusion processes in the solar interior, it may be possible to use helioseismology to constrain opacities to within a few percent throughout the solar interior.

\section{CONCLUSIONS}

The observed oscillation modes that propagate through the solar interior provide an excellent test of the physics used in solar models. Opacity differences of only a few percent have a significant effect on solar structure as inferred from solar oscillations. At this level, the fineness of the opacity table grid and interpolation errors can dominate differences in results, and accurate interpolation becomes critical. The helioseismic tests performed in this paper show that the recent Los Alamos LEDCOP opacities (http://www.t4.lanl.gov) can produce solar models that agree with the current helioseismic constraints nearly as well as models produced with the Lawrence Livermore OPAL opacities. Both of these opacity sets produce much better agreement with helioseismic data than the old Los Alamos Opacity Library [31] tables, for which the opacities were too low by as much as $20 \%$ for conditions below the solar convection zone. We strongly recommend the use of the most recent LEDCOP or OPAL opacity set for astrophysical applications. The solar oscillation data indicate that agreement would be improved by further opacity increases of up to several percent below the solar convection zone. However, the solar element abundances and solar radius must be more accurately determined, and processes such as diffusive element settling and mixing below the convection zone must be better understood before we can conclude that such an opacity increase is warranted.

\section{ACKNOWLEDGMENTS}

This work was supported in part by NASA Astrophysics Theory Program grant S30934-F. We made use of NASA's Astrophysics Data System Abstract Service and the OPAL web site http://www-phys.1lnl.gov/Research/OPAL/index.html. We are grateful to Carlos Iglesias for providing us with results in advance of publication. This work was performed under the auspices of the U.S. Department of Energy by the University of California Los Alamos National Laboratory under contract W-7405ENG-36.

\section{REFERENCES}

1. Magee, N.H., Jr., et al., Astrophysical Applications of Powerful New Databases, eds. S.J. Adelman and W.L. Wiese, San Francisco, ASP, ASP Conf. Ser. Vol. 78, 1995, p. 51; http://www.t4.lanl.gov

2. Iglesias, C.A. and Rogers, F.J., Astrophys. J. 484, 943 (1996).

3. Neuforge-Verheecke, C., Guzik, J.A., Keady, J.J., Magee, N.H., Jr., Bradley, P.A., and Noels, A., Astrophys. J. 561, 450 (2001).

4. Gough, D.O., et al., Science 372, 1281-1283; Gough, D.O., et al., Science 372, 1296-1300; Christensen-Dalsgaard, J., et al., Science 372, 1286-1290 (1996).

5. Cox, A.N., Guzik, J.A., and Kidman, R.B., Astrophys. J. 342, 1187 (1989). 
6. Gabriel, M., Astron. Astrophys. 327, 771 (1997).

7. Cohen, E.R. and Taylor, B.N., in Codata Bulletin 63, Boulder, NBS, 1986, p. 1.

8. Guenther, D.B., Demarque, P., Kim, Y.-C., and Pinsonneault, M.H., Astrophys. J. 387, 372 (1992).

9. Grevesse, N., and Noels, A, in Origin and Evolution of the Elements, eds. N. Prantzos, E. VangioniFlam, and M. Cassé , Cambridge, Cambridge Univ. Press, 1993, p. 15.

10. Iben, I., Jr., Astrophys. J. 138, 452 (1963); Astrophys. J. 141, 993 (1965); Astrophys. J. 142,421 (1965).

11. Guzik, J.A. and Swenson, F.J., Astrophys. J. 491, 967 (1997).

12. Neuforge-Verheecke, C., Goriely, S., Guzik, J.A., Swenson, F.J., and Bradley, P.A., Astrophys. J. 550, 493 (2001).

13. Burgers, J.M., Flow Equations for Composite Gases, New York, Academic, 1969.

14. Alexander, D.R. and Ferguson, J.W., private communication (1995).

15. Angulo, C., et al., Nucl. Phys. A. 656, 3 (1999).

16. Cox, J.P. and Giuli, R.T., Principles of Stellar Structure, New York, Gordon and Breach, 1968.

17. Basu, S., in Sounding Solar and Stellar Interiors, IAU Symp. 181, eds. J. Provost and F.X. Schmider, Dordrecht, Kluwer Academic Publishers, 1997, p. 137; Basu, S., Mon. Not. Roy. Astron. Soc. 298, 543 (1998).

18. Basu, S., Bahcall, J.N., and Pinsonneault, M.H, Astrophys. J. 529, 1084 (2000).

19. Pesnell, W.D., Astrophys. J. 363, 227 (1990).

20. Schou, J., \& Tomczyk, S., m2 table, http://www.hao.ucar.edu/public/research/mlso/LowL/data.html, 1996.

21. Guzik, J.A., in Proceedings of the SOHO 6/GONG 98 Workshop, 'Structure and Dynamics of the Interior of the Sun and Sun-like Stars, 'ESA SP-418, 1998, pp. 417-425.

22. Brown, T.M. and Christensen-Dalsgaard, J., Astrophys. J. 500, L195 (1998).

23. Rickert, A., Journal of Quantitative Spectroscopy and Radiative Transfer 54, 325 (1995).

24. Rose, S. J., Journal of Quantitative Spectroscopy and Radiative Transfer, 71, 635-638 (2001).

25. Turcotte, S. and Christensen-Dalsgaard, J., in Proceedings of the SOHO 6/GONG 98 Workshop, 'Structure and Dynamics of the Interior of the Sun and Sun-like Stars, 'ESA SP-418, 1998, p. 561.

26. Christensen-Dalsgaard, J., et al., in Variable Stars as Essential Astrophysical Tools, ed. C. Ibanoglu, Netherlands, Kluwer Academic, 2000, pp. 59-167.

27. Turck-Chieze, S., Space Sci. Rev. 85, 125 (1998); Tripathy, S.C., Basu, S., and ChristensenDalsgaard, J., in Poster Volume: Sounding Solar and Stellar Interiors, IAU Symp. 181, eds. J. Provost and F.X. Schmider, U. Nice, 1997, pp. 129-130.

28. Grevesse, N. and Sauval, A.J., Spa. Sci. Rev. 85, 161 (1998).

29. Brun, A.S., Turck-Chieze, S., and Zahn, J.P., Astrophys. J. 525, 1032 (1999).

30.Turcotte, S., Richer, J., Michaud, G., Iglesias, C.A., and Rogers, F.J., Astrophys. J. 504, 539 (1998).

31. Huebner, W.F., Merts, A.L., Magee, N.H. and Argo, M.F., Los Alamos Scientific Report LA-6760M, 1977. 